\title{
Genomic Characterisation of Campylobacter jejuni Isolates Recovered During Commercial Broiler Production
}

OPEN ACCESS

Edited by:

Adolfo J. Martinez-Rodriguez, Institute of Food Science Research

(CIAL), Spain

Reviewed by: Mohamed Elhadidy, University of Science and Technology at Zewail City, Egypt Nicolae Corcionivoschi, Agri-Food and Biosciences Institute (AFBI), United Kingdom

*Correspondence: Declan J. Bolton declan.bolton@teagasc.ie

Specialty section: This article was submitted to Food Microbiology,

a section of the journal

Frontiers in Microbiology

Received: 28 May 2021

Accepted: 23 July 2021 Published: 14 October 2021

Citation:

Truccollo B, Whyte $P$, Burgess CM and Bolton DJ (2021) Genomic Characterisation of

Campylobacter jejuni Isolates Recovered During Commercial Broiler Production.

Front. Microbiol. 12:716182. doi: 10.3389/fmicb.2021.716182

\author{
Brendha Truccollo, ${ }^{1,2}$ Paul Whyte ${ }^{2}$, Catherine M. Burgess' ${ }^{1}$ and Declan J. Bolton ${ }^{1 *}$ \\ ${ }^{1}$ Food Safety Department, Teagasc Food Research Centre, Dublin, Ireland, ${ }^{2}$ School of Veterinary Medicine, University \\ College Dublin, Dublin, Ireland
}

Background: Campylobacter is commonly transmitted to humans from chickens. Campylobacter jejuni is the species most frequently associated with human illness, and the most prevalent species recovered from poultry.

Objective: The objective of this study was to analyse a sub-population of $C$. jejuni from two broiler flocks on the farm and at slaughter using whole-genome sequencing to gain insights into the changes in the Campylobacter population during broiler production, including changes in virulence and antimicrobial resistance profiles.

Methods: In this study, ten composite faecal samples $(n=10)$, obtained by pooling ten fresh faecal samples $(n=10)$, were collected in the broiler house on two farms on days $14,21,28$, and $34(n=80)$ and ten composite $(n=10)$ caecal samples were collected the time of slaughter for each flock $(n=20)$. These were tested for $C$. jejuni using the ISO 10272-2:2016 method. Seven isolates were randomly selected from each of the nine Campylobacter-positive sampling points $(n=63)$ and were subjected to antimicrobial susceptibility tests. Their genomes were sequenced and the data obtained was used to characterise the population structure, virulence, antimicrobial resistance determinants and inter-strain variation.

Results: The Farm 1 isolates had three MLST types (ST257-257, ST814-661 and ST48-48) while those on Farm 2 were ST6209-464 and ST9401. Interestingly, only the MLST types positive for most of the virulence genes tested in this study persisted throughout the production cycle, and the detection of antimicrobial resistance determinants (gyrA T86I and tetO) increased after thinning and at slaughter, with the detection of new strains.

Conclusion: The persistence of the most virulent strains detected in this study throughout the production cycle has important implications for the risk to consumers and requires further investigation. The detection of new strains within the population corresponding with the time of thinning and transportation reflects previous reports and provides further evidence that these activities pose a risk of introducing new Campylobacter strains to broiler batches.

Keywords: Campylobacter, broilers, thinning, genomic characterisation, MLST, virulence genes, antimicrobial resistance 


\section{INTRODUCTION}

Campylobacter is the most common cause of bacterial foodborne illness and it is most frequently transmitted to humans from poultry, causing over 200,000 reported cases in the European Union annually (EFSA, 2019). Most infections are self-limiting and symptoms of enteritis can range from mild and asymptomatic to severe, generally first appearing within $48 \mathrm{~h}$ of ingestion and subsiding after 7 to 10 days without medical intervention (Blaser and Engberg, 2008; Kaakoush et al., 2015). More serious complications can emerge in rare cases, including the development of Guillain-Barré Syndrome, Miller Fisher Syndrome, reactive arthritis or bacteraemia (Kaakoush et al., 2015). Campylobacter is generally detected in broilers after 14 days of production, frequently reaching levels over $10^{10} \mathrm{CFU} / \mathrm{g}$ in the broiler caecum and persists until slaughter, where consumer exposure can compromise public health (Callicott et al., 2006; Hermans et al., 2011).

Previous studies document the persistence, prevalence, virulence and antimicrobial resistance of $C$. jejuni in poultry and human isolates (Desmonts et al., 2004; Talukder et al., 2008; Frazão et al., 2017; EFSA, 2019); however, there are few reports that investigate these parameters in C. jejuni throughout the broiler production cycle. Increasing resistance to antimicrobials of clinical relevance, including macrolides and fluoroquinolones, has been reported in several countries (Liu et al., 2019; Ocejo et al., 2019). The acquisition of antimicrobial resistance determinants can occur as a result of spontaneous mutations or due to horizontal gene transfer, facilitated in environments that favour C. jejuni growth, such as broiler farms, particularly in the presence of selective pressure when antimicrobial administration is required (Davies and Wales, 2019). Additionally, the manifestation of campylobacteriosis in humans is dependent on the virulence of the infecting strain and on the health of the host. Although Campylobacter lacks classical virulence factors, such as endoor enterotoxins, colonisation and survival determinants associated with motility, adhesion, invasion, capsule and biofilm synthesis, cytolethal distending toxin synthesis, molecular mimicry and secretion systems can result in the manifestation of more severe illness and persistence in the environment (Bolton, 2015; Stetsenko and Efimochkina, 2018; Wysok et al., 2020). Therefore, a more in-depth understanding of the changes in strains, virulence and antimicrobial resistance during broiler production would facilitate a more accurate assessment of the risk to public health associated with the broiler rearing cycle.

Previous studies have reported increasing antimicrobial resistance trends, and recently, the detection of more aerotolerant C. jejuni isolates in the latter stages of the production cycle has also been reported (Oh et al., 2015; Yao et al., 2016; Mouftah et al., 2021). Additionally, increased motility has been observed following C. jejuni passage through an animal host (Sang et al., 1989; Kim et al., 2012). These findings suggest that host interactions and persistence in key environments may contribute to the selection of strains with greater pathogenicity for humans.
The objective of this study was to perform a genomic characterisation of the Campylobacter population during broiler rearing, including the occurrence of virulence and antimicrobial resistance determinants.

\section{MATERIALS AND METHODS}

\section{Sample Collection}

Two intensive broiler farms $(n=2)$ were selected for this study, which was carried out between June and August 2018 (Farm 1) and between February and April 2020 (Farm 2). Both farms were located in county Monaghan in the northeast of Ireland. Each broiler house stocked approximately 30,000 broilers, Gallus gallus domesticus (Ross breed). Cattle farms were adjacent to both broiler houses. Faecal samples were collected on days $12,21,28$ and 34 on both farms and caecal samples were collected in the abattoirs, after slaughter (day 38 for Farm 1 and day 40 for Farm 2). Thinning or partial depopulation occurred on day 30 on both farms. The faecal samples consisting of ten pooled samples containing ten fresh droppings each $(n=100)$ were collected randomly using sterile scoops and sterile jars. The samples were transported to the laboratory in a cool, insulated carrier box and were processed within $4 \mathrm{~h}$ of collection. On the day of slaughter, caecal samples consisting of ten pooled samples containing ten caeca each $(n=100)$ were randomly collected from the slaughterhouse, transported in a cool box and processed within $24 \mathrm{~h}$.

\section{Campylobacter Spp. Enumeration}

Enumeration was carried out in accordance with ISO 102722:2016. To $25 \mathrm{~g}$ of each pooled faecal or caecal sample, $225 \mathrm{ml}$ of Bolton Broth (Oxoid, Basingstoke, United Kingdom) was added. Samples were stomached for $60 \mathrm{~s}$ and serially diluted 10 -fold in maximum recovery diluent (MRD, Oxoid, Basingstoke, United Kingdom). Each dilution was spread on modified charcoal cefoperazone deoxycholate agar (mCCDA, Oxoid, Basingstoke, United Kingdom) and incubated at $42^{\circ} \mathrm{C}$ microaerobically $\left(10 \% \mathrm{CO}_{2}, 5 \% \mathrm{O}_{2}\right.$ and balancing $\left.\mathrm{N}_{2}\right)$ in a controlled atmosphere incubator (MACS VA-500, Don Whitley) for $48 \mathrm{~h}$.

\section{Confirmatory Tests}

Five randomly selected presumptive colonies $(n=5)$ were sub-cultured from each sample on Mueller Hinton agar (MHA, Oxoid, Basingstoke, United Kingdom) supplemented with 5\% defibrinated horse blood and incubated at $42^{\circ} \mathrm{C}$ microaerobically for $48 \mathrm{~h}$. From each MHA culture, 2-3 colonies were inoculated into $2 \mathrm{ml}$ of Mueller Hinton broth (MHB; Oxoid, Basingstoke, United Kingdom) and incubated at $42^{\circ} \mathrm{C}$ microaerobically for $48 \mathrm{~h}$ and DNA was subsequently extracted using the Qiagen DNeasy Blood and Tissue kit (Qiagen, Manchester, United Kingdom) following the manufacturer's instructions. The species of each isolate was confirmed by PCR using a previously published method (Wang et al., 2002). The remaining 
growth on MHA was harvested into $1-2 \mathrm{ml}$ of defibrinated horse blood and stored at $-80^{\circ} \mathrm{C}$ for future analyses.

\section{Antimicrobial Susceptibility}

Antimicrobial susceptibility tests were carried out on 63 isolates in total $(n=63)$, constituting seven randomly selected isolates $(n=7)$ from each Campylobacter positive sampling point at each farm $(n=49)$ and abattoir $(n=14)$ using the broth microdilution method as per ISO 20776-1:2006 and EUCAST recommendations. Susceptibility to erythromycin $(1-512 \mathrm{mg} / \mathrm{l})$, ertapenem $(0.125-4 \mathrm{mg} / \mathrm{l})$, tetracycline (TET; $0.5-64 \mathrm{mg} / \mathrm{l}$ ), ciprofloxacin (CIP; $0.125-32 \mathrm{mg} / \mathrm{l})$, gentamicin $(0.5-16 \mathrm{mg} / \mathrm{l})$ and chloramphenicol $(2-64 \mathrm{mg} / \mathrm{l})$ was interpreted using EUCAST guidelines in accordance with ISO 20776-1:2006. Each isolate was grown in $10 \mathrm{ml}$ of $\mathrm{MHB}$, which was incubated overnight at $42^{\circ} \mathrm{C}$ microaerobically. The inoculum was diluted 10 -fold in $10 \mathrm{ml}$ of $\mathrm{MH}-\mathrm{F}$ broth (clarified cation adjusted MHB with $20 \mathrm{mg} / 1 \quad \beta$-NAD and 5\% lysed horse blood) to yield a solution with $1 \times 10^{6} \mathrm{CFU} / \mathrm{ml}$. Fifty microlitres of this solution was added to each well containing $50 \mu \mathrm{l}$ broth containing double the desired antimicrobial concentration to yield a final inoculum of $5 \times 10^{5} \mathrm{CFU} / \mathrm{ml}$. C. jejuni ATCC 33560 and Staphylococcus aureus ATCC 22391 were used as controls as per EUCAST recommendations. C. jejuni plates were incubated at $42^{\circ} \mathrm{C}$ microaerobically for $24-48 \mathrm{~h}$. S. aureus was incubated at $37^{\circ} \mathrm{C}$ aerobically for $24 \mathrm{~h}$. Resistance and susceptibility were determined using epidemiological cut-off points in accordance with EFSA recommendations (Aerts et al., 2019).

\section{Whole-Genome Sequencing}

The same isolates as above $(n=63)$ from Farms 1 and 2 were selected for genomic characterisation. Single colonies from mCCDA were sub-cultured into MHA and incubated at $42^{\circ} \mathrm{C}$ microaerobically for $48 \mathrm{~h}$. A loopful from each culture was suspended in PBS, gently mixed and centrifuged at $5000 \times g$ for $5 \mathrm{~min}$. The supernatant was discarded and $180 \mu \mathrm{l}$ of buffer ATL and $20 \mu$ of Proteinase K were added to each pellet. The remaining steps of the DNA extraction were followed per the manufacturer's instructions (DNA Blood and Tissue Kit, Qiagen, Manchester, United Kingdom). RNA contamination was removed by incubating the samples at $37^{\circ} \mathrm{C}$ for $30 \mathrm{~min}$ with $10 \mu \mathrm{l}$ of $100 \mathrm{mg} / \mathrm{ml}$ RNase A (Thermo Fisher, Paisley, United Kingdom) after cell lysis, then returning the samples to $57^{\circ} \mathrm{C}$ for $30 \mathrm{~min}$. Each resulting DNA sample was quantified using the BR dsDNA kit with the Qubit 4.0 fluorometer (Thermo Fisher, Paisley, United Kingdom). Further quantification and 260:280 and 260:230 ratios were measured using the NanoDrop 2.0. Lastly, each sample was run on $1.0 \%$ agarose gel in $1 \mathrm{X}$ TBE buffer for $40 \mathrm{~min}$ at $100 \mathrm{~V}$ to check for DNA degradation. Samples with more than 500 ng of DNA, free from RNA contamination and with a $260: 280$ ratio between 1.8 and 2 were subject to paired-end $150 \mathrm{bp}$ whole-genome sequencing on the Illumina NovaSeq 6,000 platform (Illumina, Cambridge, United Kingdom) with 100x coverage (Novogene, Cambridge, United Kingdom). Library preparation was performed by Novogene, Cambridge, United Kingdom, as per standard protocol using the NEBNext
DNA Library Prep kit (New England Biolabs, Massachusetts, United States).

\section{Bioinformatic Analysis}

The trimmed reads provided were assembled on SPades v. 3.14 (Bankevich et al., 2012) with the - careful parameter. Assembly quality was checked using QUAST (Gurevich et al., 2013) using C. jejuni NCTC 11168 as a reference. They were annotated on Prokka v. 2.14 (Seemann, 2014) with - use genus and genus Campylobacter parameters.

The gff outputs from annotation were analysed in Roary (Page et al., 2015). The core genome alignment file output from Roary was used to construct a maximum likelihood tree using RAXML with m GTRGAMMA with 500 bootstraps (Stamatakis, 2014). Roary was also used to compare differences in the genes present in each strain using the query pan_genome command with - a difference, and differences in the genes present within each strain between sampling time points using the same command. The nucleotide sequences from genes and coding sequences (CDS) found to be uniquely present in a strain were extracted from pan_genome_reference.fa using query_pan_genome - a gene_multifasta. The outputs from strain vs. strain analyses were concatenated and submitted to eggNOG mapper v. 5.0 for functional annotation with a taxonomic scope restricted to Epsilonproteobacteria and standard parameters (Huerta-Cepas et al., 2017).

Multilocus sequence typing (MLST), which targets seven C. jejuni housekeeping genes, was carried out for each isolate using MLST ${ }^{1}$ equipped with the PubMLST database (Jolley et al., 2018). cgMLST, which targets of 1,300 core genes in C. jejuni, was also carried out to further distinguish the genomes analysed in this study. cgMLST alleles were first determined using cgMLST and $\operatorname{cgSTs}$ were determined using $\operatorname{cgMLST}^{2}$ Finder v. 1.1 (Clausen et al., 2018).

Abricate equipped with VFDB and Victors was used to extract all virulence genes from each isolate (Chen et al., 2016; Sayers et al., 2019). It was then equipped with Resfinder v. 4.1, CARD, ARG-ANNOT and NCBI AMR Finder to identify antimicrobial resistance determinants in each isolate (Gupta et al., 2014; Jia et al., 2017; Feldgarden et al., 2019). Hits with less than $80 \%$ identity and/or coverage were filtered out of the analysis. ${ }^{3}$

Assemblies were filtered to remove contigs $<200 \mathrm{bp}$ using BBMap v. 38.22 (Bushnell, 2014) prior to submitting each genome into BioProject ID PRJNA688841.

\section{Statistical Analysis}

Significant differences in the proportions of COG (Clusters of Orthologous Groups) categories between strains were calculated using a chi-square test in R Studio (Boston, MA, United States) equipped with $\mathrm{R}$ v. 3.6.3.

\footnotetext{
${ }^{1}$ https://github.com/tseemann/mlst

${ }^{2}$ https://github.com/aldertzomer/cgmlst

${ }^{3}$ https://github.com/tseemann/abricate
} 


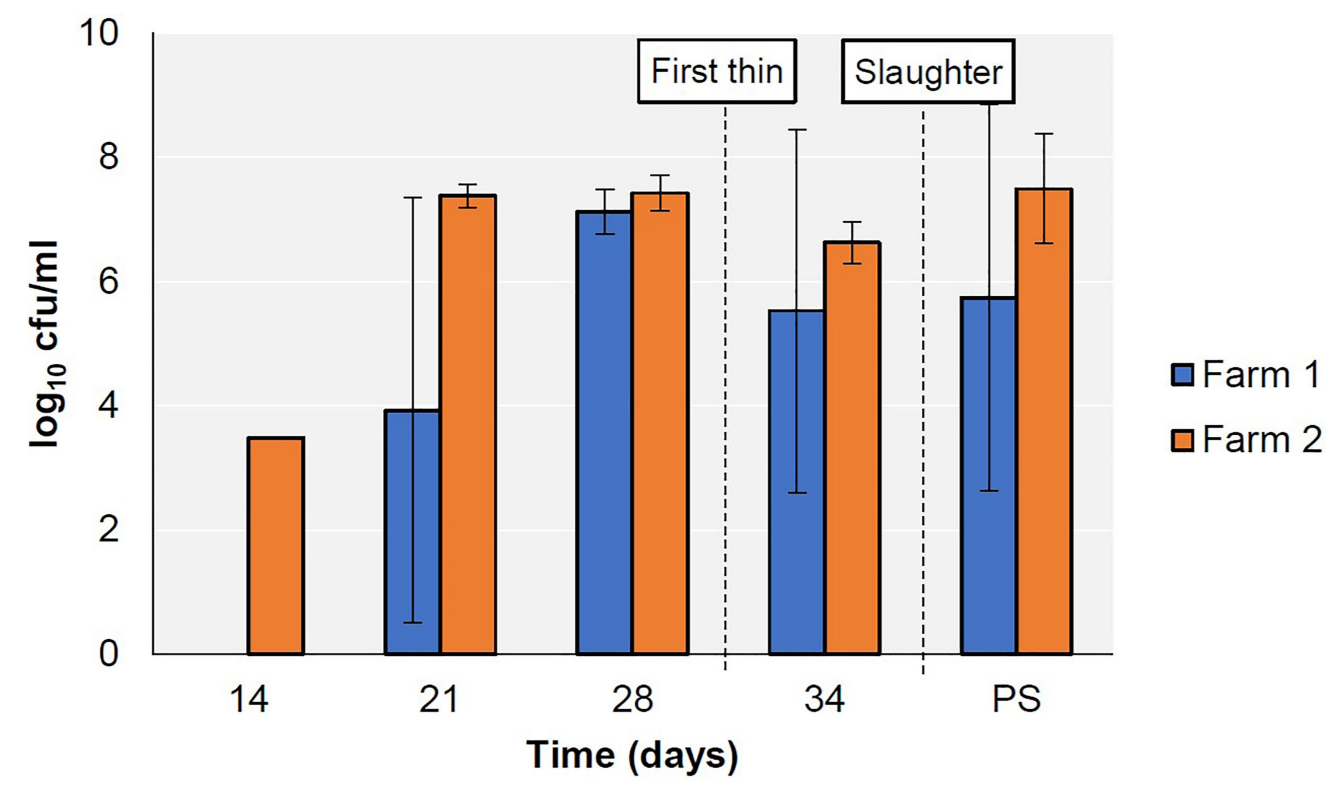

FIGURE 1 | Prevalence of Campylobacter spp. in faecal (days 14-34) and caecal (PS; post-slaughter) samples from two broiler farms.

\section{RESULTS}

\section{Prevalence}

Campylobacter was first detected on Farm 1 on day 21 and on Farm 2 on day 14, persisted on both farms until the end of the production cycle (Figure 1), and was subsequently detected in the abattoir caecal samples. All Campylobacter isolates detected in this study were confirmed as C. jejuni.

\section{Genome Analysis}

A total of sixty-three genomes were sequenced ( $n=63)$ : twentyeight from Farm 1 from days 21, 28 and 34 during the production cycle and the abattoir caecal samples $(n=28)$ and thirty-five from Farm 2 from days 14, 21, 28 and 34 of the production cycle and the abattoir samples $(n=35)$. Genomes were assembled with 27 contigs on average, ranging from 14 to 60 . Genome lengths ranged from 1.6 to $1.8 \mathrm{Mbp}, \mathrm{GC}$ content was on average $30.3 \%$, ranging from 30.07 to $30.47 \%$. The genomes were assigned to five MLST types and six cgST types, and their mean number of base pairs is provided in Table 1.

\section{Population Structure}

A maximum likelihood (ML) tree was constructed using the core genome alignment of all isolates to analyse the resulting population structure (Figure 2). Clusters corresponding to each MLST type identified were detected with very short distances between genomes (Figure 2), indicating the possibility of clonal expansion. Notably, two cgST types were detected within the sequence type 48 clonal complex 48 (ST48-48) cluster, which were not evident in the ML tree (cgST-34272
TABLE 1 | Average genome length per MLST and cgST type.

\begin{tabular}{lccc}
\hline Source & MLST Type & MLST Type & $\begin{array}{c}\text { Average length } \\
\text { (bp; mean } \pm \text { SD) }\end{array}$ \\
\hline \multirow{2}{*}{ Farm 1 } & ST48-48 $(n=14)$ & 34286 & $1,641,757.25 \pm 579.77$ \\
& ST257-257 $(n=11)$ & 12407 & $1,688,819.18 \pm 1.54$ \\
& ST814-661 $(n=3)$ & 10385 & $1,823,975.67 \pm 2,119.17$ \\
\hline \multirow{2}{*}{ Farm 1 } & ST6209-464 $(n=32)$ & 21553 & $1,770,576.47 \pm 616.14$ \\
& ST9401 $(n=3)$ & 16486 & $1,769,724.00 \pm 473.68$ \\
\hline
\end{tabular}

and cgST-34286; Figure 2), as the corresponding alleles may not have comprised part of the core genome alignment between all strains.

\section{MLST/CgMLST}

Three MLST types were detected in Farm 1 along with four cgST types, while two MLST types were detected on Farm 2 (Figure 3). The breakdown of alleles and profiles is provided in Appendix S1.

On Farm 1, ST257-257 (cgST-12407) was the only sequence type detected on day 21 (Figure 3). Subsequently, ST257257 and ST48-48 were both detected on day 28. By day 34, only ST48-48 was detected, and additionally, ST814-661 was detected for the first time. These two strains persisted until slaughter on day 38. Furthermore, two cgSTs also belonging to ST48-48 were detected on days 28 and 38 cgST-34272 and cgST-34286.

On Farm 2, the only sequence type detected during the rearing period between days 14 and 34 was ST6209-464 (cgST21553; Figure 3). This strain persisted until slaughter on day 
Tree scale: 0.01

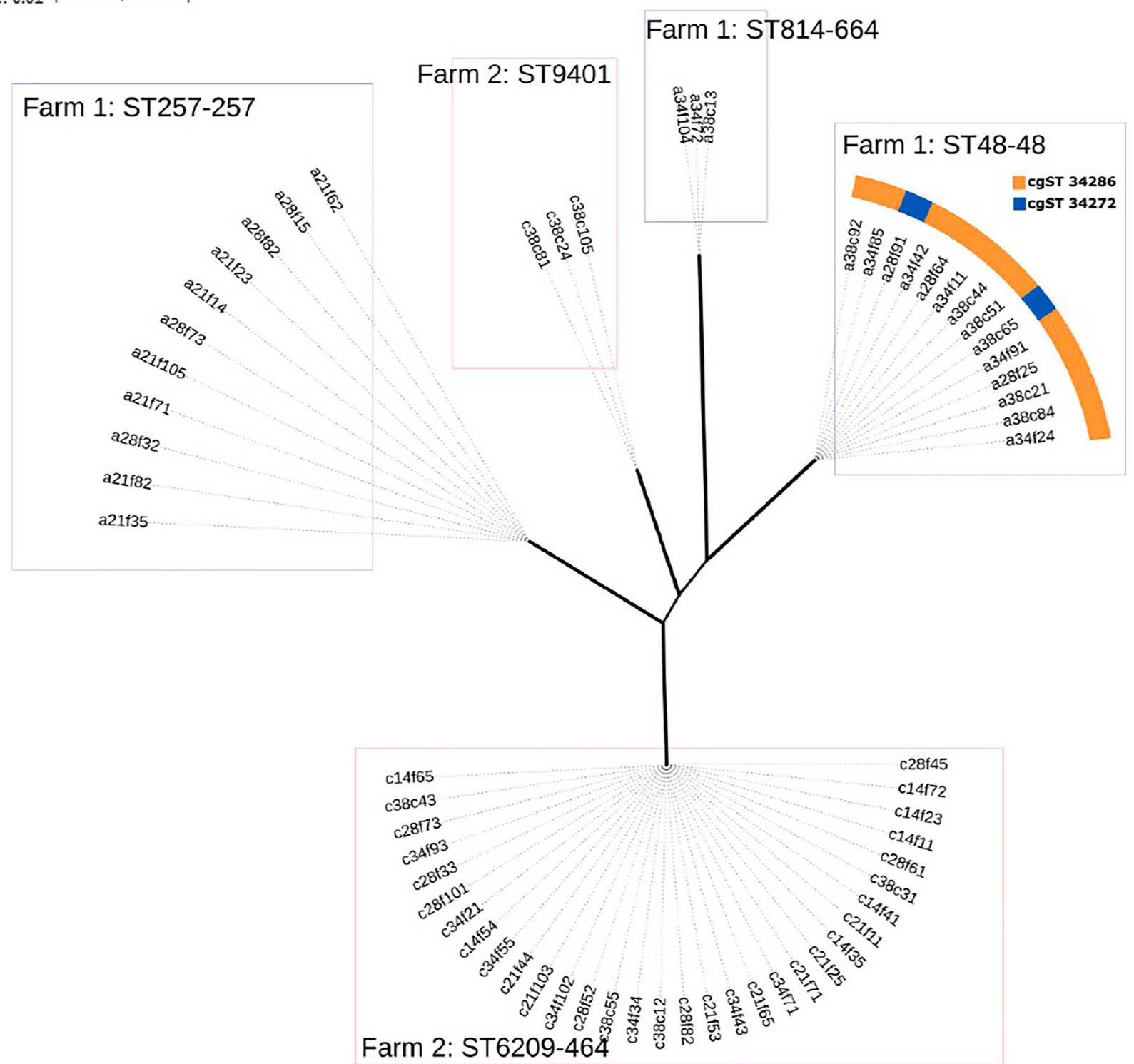

FIGURE 2 | Maximum likelihood (ML) tree of the core genome alignments of isolates from Farm 1 and Farm 2 with 500 bootstraps. Branch width corresponds to bootstrap values, where wider branches correspond to higher bootstrap values that ranged from 66 to 100 between MLST types. Genomes are named to indicate farm $(a=1$ and $c=2)$, broiler age (days), sample type ( $f=$ faecal and $c=$ caecal), sample number ( 1 to 10$)$ and colony number ( 1 to 5$)$, i.e. 'a21f62'.

40, when ST9401 (cgST-16486) was also detected for the first time.

\section{Whole-Genome Comparison Between MLST Types}

A whole-genome comparison between the MLST types detected in this study was performed and is provided in Appendix S2 along with a full list of the genes that were detected and their respective individual functional annotation.

The three MLST types detected on Farm 1 (ST257-257, ST48-48 and ST814-661) were analysed. The genes that were present in one MLST type but absent from the others were submitted for functional annotation to characterise the functional breakdown of the unique accessory genome in each of these isolates. ST814-661 was significantly associated with genes involved in cell cycle control $(p<0.01)$; replication, recombination and repair $(p<0.001)$; and intracellular trafficking, secretion and vesicular transport $(p=0.001)$ compared to ST257-257. It was also significantly associated with a higher prevalence of genes involved in transcription $(p<0.05)$; replication, recombination and repair $(p<0.01)$; and intracellular trafficking, and secretion and vesicular transport $(p<0.01)$ compared to ST48-48, while ST48-48 carried genes involved in inorganic ion transport and metabolism $(p<0.05)$, which were not present in ST814-661. 


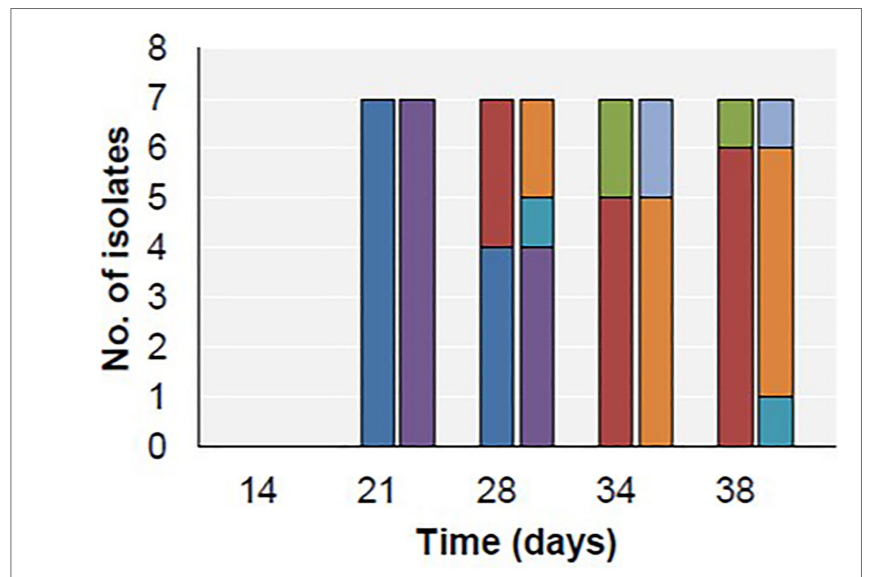

घST257-257 घST48-48 घST814-664 घcgST 12407 घcgST $34272 \square$ cgST 34286 口cgST 10385

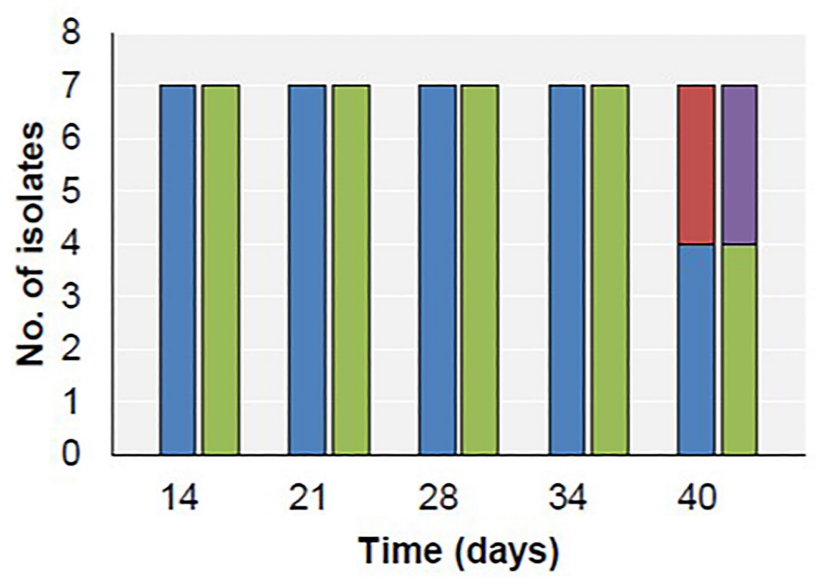

口ST6209-464 घST9401 口cgST 21553 घcgST 16486

FIGURE 3 | Distribution of MLST (first bar at each time point) and cgST types (second bar at each time point) in Farm 1 (top) and Farm 2 (bottom).

On Farm 2, a higher prevalence of genes involved in intracellular trafficking, secretion and vesicular transport $(p<0.05)$ was associated with the unique accessory genome of ST6209464 compared to ST9401, while no significant difference was detected in the remaining COG categories.

\section{Virulence Gene Prevalence}

In total, 153 virulence genes were detected in the 63 genomes analysed in this study (Appendix S3). Of these, $72.5 \%$ were present in all 63 genomes, which are associated with functions including adhesion, invasion, cytotoxin production, motility, chemotaxis, lipooligosaccharide synthesis, capsule synthesis, biofilm formation and colonisation. The genes that were present on Farm 1 with a prevalence ranging between 1 and 99\% are shown in Figure 4.

On this farm, the prevalence of genes associated with adherence, biofilm formation, capsule, colonisation, invasion, motility and type IV secretion systems (T4SS) increased from day 21 to day 38, which corresponded with the detection of ST48-48 and ST-814-661. Interestingly, genes associated with capsule synthesis $g m h A 2$, $h d d A, h d d C$ and Cj0618, which may be associated with colonisation, were more prevalent on days 21 and 28, when ST257-257 was still detected and was less frequently found on days 34 and 38 with the recovery of ST814-661. In contrast, T4SS genes were detected on days 34 and 38 in ST814-661.

On Farm 2, no differences were observed in the prevalence of virulence genes during the broiler sampling period (days 14-34), as only one MLST type genome was detected (ST6209-464). With the exception of the absence of the capsule synthesis genes Cj1421c and Cj1422c on day 14 from one strain, the other genes were all present during the broiler production cycle. Interestingly, the prevalence of genes associated with capsule synthesis (Cj1421c, Cj1422c, Cj1426c, Cj1427c, Cj1435c, Cj1436c, Cj1437c, Cj1440c, fcl, glf, gmhA2, hddA, hddC, $k f i D$ and $r f b C$ ), motility (pseD/maf2) and T4SS (Cjp54/virB7, $\operatorname{virB10}$, virB11, virB4, virB8, virB9 and virD4) decreased on day 38, which corresponded to the detection of ST9401. Capsule synthesis genes Cj1421c and Cj1422c were absent from one ST6209-464 isolate on day 14 and from two isolates on day 38. Additionally, two out of three ST9401 isolates carried the capsule synthesis gene $f c l$. Overall, no change in the prevalence of virulence genes was observed during the broiler rearing period in Farm 2; however, a decrease in these virulence genes was observed with the detection of ST9401 at slaughter.

\section{Antimicrobial Resistance}

Antimicrobial resistance was measured phenotypically, and the prevalence of genomic antimicrobial resistance determinants was characterised for each isolate (Figure 4).

Antimicrobial resistance was not detected in isolates from Farm 1 until day 34, with the isolation of ST814-661. On day 34 , resistance to TET was detected in one isolate, while on day 38, one ST814-661 isolate was phenotypically resistant to both TET and CIP.

In contrast, on Farm 2, all isolates detected during the broiler rearing period exhibited resistance to TET (ST6209464), while on day 40, a decrease in the prevalence of TET resistance was observed, with the detection of TET ST9401, which was susceptible to TET. Furthermore, two ST9401 isolates recovered on day 40 exhibited phenotypic resistance to CIP. All strains except ST814-661 carried cmeABC and $c m e R$, which encodes the multidrug efflux system (Figure 4).

There was complete agreement between the prevalence of the TET resistance gene tet $O$ and TET resistance in ST6209, while a higher recovery rate of tetO was detected in ST814661 compared to that observed in phenotypic susceptibility tests. Similarly, the fluoroquinolone resistance amino acid substitution gyrA T86I was more prevalent than phenotypic resistance to CIP, as it was found in all ST814-661 and ST9401 


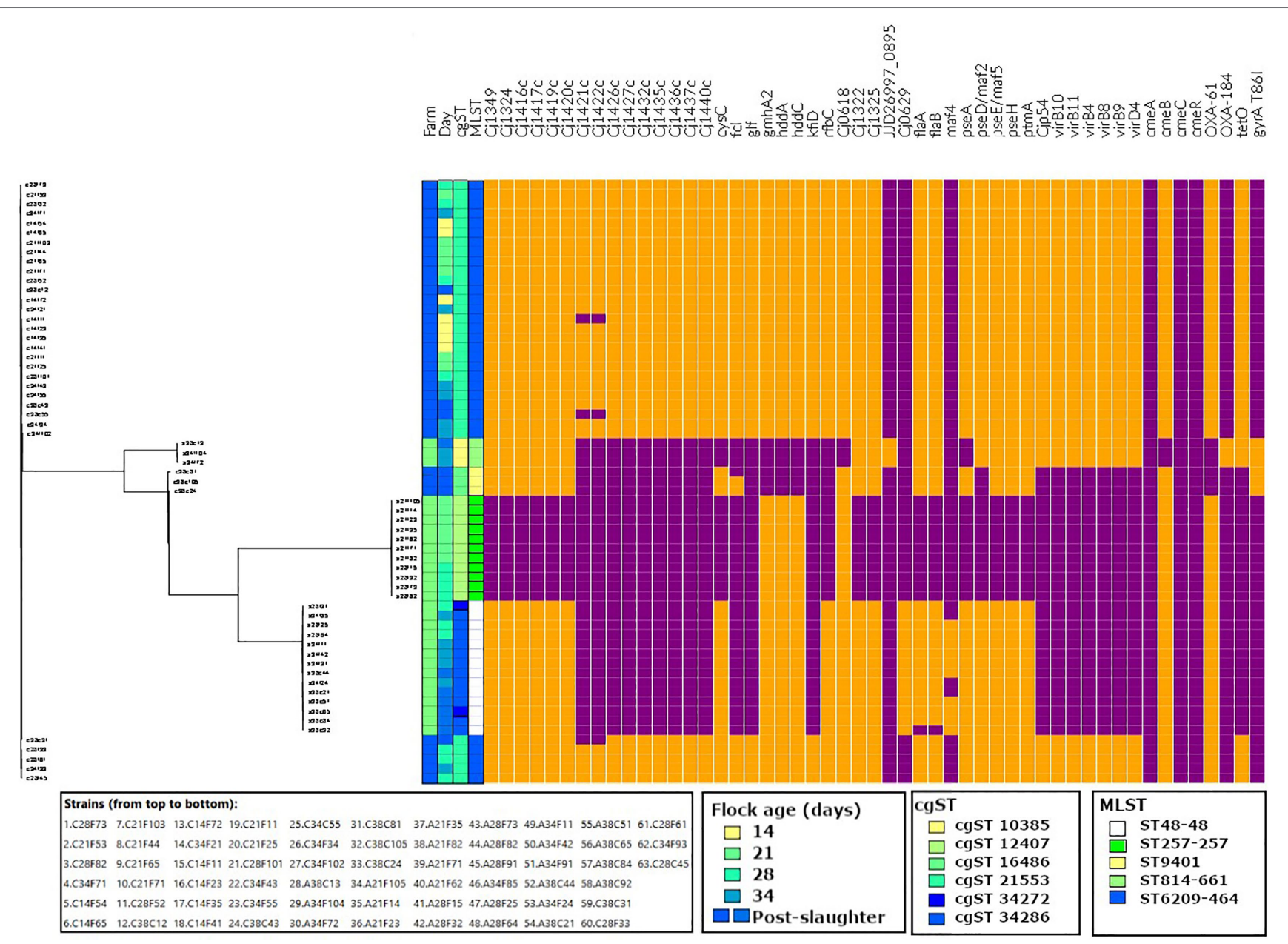

FIGURE 4 | Virulence (Cj1349 - virD4) and antimicrobial resistance (cmeA - gyrA T86l) profiles of the strains isolated in this study, aligned to the tree output from Roary. Data visualised on Phandango (http://jameshadfield. github.io/phandango/\#/). Farm 1: green; Farm 2: blue. Present: orange; absent: purple.

isolates, as compared to one out of three ST814-661 and two out of three ST9401 that exhibited resistance phenotypically. Additionally, isolates that carried tetO but did not exhibit phenotypic resistance showed reduced susceptibility as compared to the isolates that were tet $O$ negative.

ST814-661 carried $c m e A, c m e C$ and $c m e R$, while $c m e B$ was detected with less than $80 \%$ identity and thus filtered out of the analysis. Additionally, all strains except ST9401 carried $b l a_{\mathrm{OXA}-61}$, which is associated with $\beta$-lactam resistance. $b l a_{\mathrm{OXA}-61}$ was the most frequently detected $b l a_{\text {OXA }}$ gene, present in ST257257, ST48-48 and ST6209, while bla $a_{\text {OXA-184 }}$ was found in ST814-661.

\section{DISCUSSION}

In this study, we analysed the progression of $C$. jejuni sequence types during rearing on two commercial broiler farms in Ireland. The genomic proximity of same-MLST type strains indicates the possibility of clonal expansion in the broiler house (Figure 1). In Farm 1, three different MLST types were identified. ST48-48 and ST814-661 persisted until slaughter, while ST257-257 was only detected on days 21 and 28. This indicates the possibility that ST257-257 may have been outcompeted by the other two strains or that it was below the level of detection of the sampling strategy applied in this study. In the PubMLST database, ST257257 and ST48-48 are most commonly recovered from humans, chicken and cattle, while ST814-661 is most commonly recovered from humans and chickens (Jolley et al., 2018), although this could reflect a submission bias. Additionally, the presence of two cgST types within ST48-48 in this study (cgST-34286 and cgST-34272) early in the production cycle indicates that two distinct types belonging to ST48-48 may have colonised this flock. Clonal complex ST-48 was previously found in a wide range of patients, including reactive arthritis patients, and often in association with fluoroquinolone resistance (Nielsen et al., 2010; Collado et al., 2018). In contrast, in 2012, it was associated with fluoroquinolone susceptibility in the United Kingdom, which was also observed in this study (Cody et al., 2012). Furthermore, clonal complex ST-257 was previously reported to be associated with hospitalisation of infected humans (Harvala et al., 2016). 
In Farm 2, ST6209-464 was found during the rearing cycle. There are only twenty-seven records of previously recovered ST6209-464 in PubMLST, all of which were from humans or chickens (Jolley et al., 2018). All ST6209-464 isolates in this study were TET resistant, which reflects a recent study that reported TET and CIP resistance is common among ST6209464 isolates in Lithuania (Aksomaitiene et al., 2019). During slaughter, ST9401 was also detected and carried the fluoroquinolone resistance determinant gyrA T86I. This MLST type has rarely been reported, twice associated with humans and once in chicken samples (Jolley et al., 2018).

Overall, two distinct patterns of Campylobacter detection were observed on Farms 1 and 2, whereby multiple MLST types were identified during the production cycle in the former and only one was found in the latter. The replacement of a dominant strain during broiler production has been previously reported, reflecting the dynamics observed on Farm 1 (Bull et al., 2006). In contrast, it has also been reported that a predominant strain can persist throughout the production cycle and after slaughter, reflecting the results observed on Farm 2 (Inglis et al., 2020). The difference in the diversity of MLST strains across the two farms could reflect individual farm practices including differences in biosecurity compliance, adjacent local environmental reservoirs including livestock and wildlife and/or antimicrobial interventions or may be a seasonal factor (Jorgensen et al., 2011; Battersby et al., 2016; Meunier et al., 2016). Indeed, Farm 2 was sampled while COVID-19 and avian influenza restrictions were operational, which may have contributed to increased biosecurity in this farm as compared to Farm 1, resulting in less opportunity for different Campylobacters to access the flock, as previously observed (DAFM, 2020; HPSC, 2020). Notably, the detection of ST814-661 in Farm 1 and ST9401 in Farm 2 samples corresponds with thinning and slaughter, respectively, which suggests that these practices may still pose a risk of contamination, as previously reported (Koolman et al., 2014; Prachantasena et al., 2016).

We detected 153 virulence genes in total in this study (Appendix S3). Most of these genes were detected in all isolates that were tested, which includes the ubiquitous presence of virulence genes associated with adhesion and invasion (ciaB, $c a d F$ and $p l d A$ ) and cytotoxin production $(c d t A B C)$. Thus, all of the isolates identified in this study have the potential to cause human illness (Konkel et al., 1999; Ziprin et al., 2001; Flanagan et al., 2009; Lai et al., 2016; Scuron et al., 2016). This is supported by evidence that isolates belonging to these MLST types have been previously isolated from humans (Jolley et al., 2018). On Farm 1, genes associated with colonisation, capsule synthesis, motility, biofilm formation, adhesion and T4SS were prevalent in isolates that persisted until the end of the broiler production cycle (ST48-48 and ST814-661). Bacterial T4SS contributes to the delivery of virulence factors into eukaryotic or prokaryotic cells. In Campylobacter spp., T4SS can contribute to the activity of adhesins and invasins, such as Campylobacter invasion antigen (Cia) proteins (Bacon et al., 2000; Sgro et al., 2019). ST6209-464, which was the only strain detected on Farm 2 during the production cycle, carried the highest number of virulence genes of any strain in this study, carrying between 148 and 150 virulence determinants, while the remaining isolates tested positive for between 114 and 133 virulence determinants. Overall, the strains with the greatest number of virulence determinants in this study persisted until the end of the production cycle (ST48-48, ST814-661 and ST6209-464).

On Farms 1 and 2, more antimicrobial resistance determinants were detected towards the end of the production cycle and at slaughter, which in Farm 1 corresponded with the detection of tetO and gyrA T86I-carrying ST814-661, and in Farm 2 corresponded with the detection of gyrA T86I-positive ST9401. Furthermore, the prevalence of phenotypic TET resistance in ST6209-464 was consistent with the detection of tet $O$, and a high level of concordance between phenotypic and genotypic resistance was also observed for tetO in ST814-661 for two of the three strains that were TET resistant. The third isolate exhibited reduced susceptibility, while gyrA T86I was detected in six strains, three belonging to ST9401 and three from ST814-661, with 50\% concordance with the observed phenotypic results. Notably, ST814-661 strains did not carry the complete $c m e B$ gene that encodes a multidrug efflux pump, which may have contributed to reduced TET and fluoroquinolone resistance, despite the presence of tet $O$ and gyr $A$ T86I (Pumbwe and Piddock, 2002). Comparable levels of gyrA T86I carriage and CIP resistance concordance have also been previously reported (Wysok et al., 2020).

While significant differences in the prevalence of genes associated with specific biological functions were identified between MLST types (Appendix S2), no COG categories were over-represented in ST48-48 as compared to ST257-257. In contrast, inorganic ion metabolism and transport were more prevalent in ST48-48 compared to ST814-661. It was previously suggested that this functional category is under selective pressure in Vibrio spp. using gene ontology enrichment analysis; however, further investigation is needed to confirm whether this category is important for the persistence of Campylobacter spp. in broilers (Rabby et al., 2015). The same study also proposes that COG categories, such as intracellular trafficking and secretion, may be under selective pressure due to their role in host-pathogen interactions and nutrition acquisition and metabolism. It has also been previously suggested that variable distributions of accessory genes between strains can be associated with ecological niche adaptation (McInerney et al., 2017), which has been observed in accessory genes from Staphylococcus haemolyticus that exhibited variable distribution across a variety of niches, including human, copper, willow and rice niches (Chaudhry and Patil, 2020). Similarly, C. jejuni carries accessory genes that are differentially distributed across strains, indicating that a similar form of ecological adaptation may occur (Park et al., 2020).

\section{CONCLUSION}

In this study, we investigated changes in $C$. jejuni populations during broiler rearing on two Irish broiler farms. We identified five MLST types and six cgSTs across the two farms. The first detection of two of the MLST types (ST814-661 and ST9401) corresponded with the time of thinning and slaughter, respectively. More antimicrobial resistance and virulence determinants were 
detected in isolates at slaughter than during the early stages of the production cycle. Pangenomic analysis revealed that the most frequently detected strains (ST48-48 and ST6209-464) carried more genes associated with COG categories including intracellular trafficking and inorganic ion metabolism and transport compared to less frequently detected strains (ST814-661 and ST9401, respectively). Overall, our results indicate a possible relationship between broiler rearing and changes in C. jejuni populations that could be of public health significance; however, further investigation with larger sample sizes, more farms and under different conditions is required to firmly establish if this observation holds true in the broiler production environment.

\section{DATA AVAILABILITY STATEMENT}

The datasets generated for this study can be found under BioProject ID PRJNA688841.

\section{AUTHOR CONTRIBUTIONS}

DB obtained the funding. All authors were responsible for conceiving and the design of the study. BT acquired, analysed, and interpreted the data. BT drafted the manuscript while $\mathrm{PW}, \mathrm{CB}$, and $\mathrm{DB}$ did the critical revision.

\section{REFERENCES}

Aerts, M., Battisti, A., Hendriksen, R., Kempf, I., Teale, C., Tenhagen, B. A., et al. (2019). Technical specifications on harmonised monitoring of antimicrobial resistance in zoonotic and indicator bacteria from food-producing animals and food. EFSA J. 17:e05709. doi: 10.2903/j.efsa.2019.5709

Aksomaitiene, J., Ramonaite, S., Tamuleviciene, E., Novoslavskij, A., Alter, T., and Malakauskas, M. (2019). Overlap of antibiotic resistant campylobacter jejuni MLST genotypes isolated From humans, broiler products, dairy cattle and wild birds in Lithuania. Front. Microbiol. 10:1377. doi: 10.3389/ fmicb.2019.01377

Bacon, D. J., Alm, R., Burr, D. H., Hu, L., Kopecko, D. J., Ewing, C. P., et al. (2000). Involvement of a plasmid in virulence of campylobacter jejuni 81-176. Infect. Immun. 68, 4384-4390. doi: 10.1128/IAI.68.8.4384-4390.2000

Bankevich, A., Nurk, S., Antipov, D., Gurevich, A. A., Dvorkin, M., Kulikov, A. S., et al. (2012). SPAdes: a new genome assembly algorithm and its applications to single-cell sequencing. J. Comput. Biol. 19, 455-477. doi: 10.1089/cmb.2012.0021

Battersby, T., Whyte, P., and Bolton, D. (2016). Protecting broilers against campylobacter infection by preventing direct contact between farm staff and broilers. Food Control 69, 346-351. doi: 10.1016/j.foodcont.2016.04.053

Blaser, MJ, and Engberg, J. (2008). Clinical Aspects of campylobacter jejuni and campylobacter coli infections. Campylobacter third edition. Am. Soc. Microbiol. 99-121. doi:10.1128/9781555815554.

Bolton, D. J. (2015). Campylobacter virulence and survival factors. Food Microbiol. 48, 99-108. doi: 10.1016/j.fm.2014.11.017

Bull, S. A., Allen, V. M., Domingue, G., Jørgensen, F., Frost, J. A., Ure, R., et al. (2006). Sources of campylobacter spp. colonizing housed broiler flocks during rearing. Appl. Environ. Microbiol. 72, 645-652. doi: 10.1128/ AEM.72.1.645-652.2006

Bushnell, B. (2014). BBMap: A Fast, Accurate, Splice-Aware Aligner. Berkeley, CA (United States): Lawrence Berkeley National Lab.(LBNL).

Callicott, K. A., Frioriksdóttir, V., Reiersen, J., Lowman, R., Bisaillon, J. R., Gunnarsson, E., et al. (2006). Lack of evidence for vertical transmission of

\section{FUNDING}

This research was funded by the Teagasc (the Agriculture and Food Development Authority for Ireland) under the Teagasc Walsh Scholarship scheme (project number 0028).

\section{ACKNOWLEDGMENTS}

We would like to thank our agricultural collaborators for facilitating sampling and access to broiler farms for this study, Leonard Koolman for providing assistance with sample collection and Amélie Rouger for providing bioinformatic analysis training.

\section{SUPPLEMENTARY MATERIAL}

The Supplementary Material for this article can be found online at: https://www.frontiersin.org/articles/10.3389/fmicb.2021.716182/ full\#supplementary-material.

APPENDIX S1 | MLST and cgMLST allelic profiles.

APPENDIX S2 | Functional annotation of differences between the gene content of MLST strains.

APPENDIX S3 | Detection of virulence and antimicrobial resistance determinants.

campylobacter spp. in chickens. Appl. Environ. Microbiol. 72, 5794-5798. doi: 10.1128/AEM.02991-05

Chaudhry, V., and Patil, P. B. (2020). Evolutionary insights into adaptation of staphylococcus haemolyticus to human and non-human niches. Genomics 112, 2052-2062. doi: 10.1016/j.ygeno.2019.11.018

Chen, L., Zheng, D., Liu, B., Yang, J., and Jin, Q. (2016). VFDB 2016: hierarchical and refined dataset for big data analysis-10 years on. Nucleic Acids Res. 44, D694-D697. doi: 10.1093/nar/gkv1239

Clausen, P. T. L. C., Aarestrup, F. M., and Lund, O. (2018). Rapid and precise alignment of raw reads against redundant databases with KMA. BMC Bioinformatics 19, 1-8. doi: 10.1186/s12859-018-2336-6

Cody, A. J., McCarthy, N. M., Wimalarathna, H. L., Colles, F. M., Clark, L., Bowler, I. C. J. W., et al. (2012). A longitudinal 6-year study of the molecular epidemiology of clinical campylobacter isolates in Oxfordshire, United Kingdom. J. Clin. Microbiol. 50, 3193-3201. doi: 10.1128/JCM.01086-12

Collado, L., Muñoz, N., Porte, L., Ochoa, S., Varela, C., and Muñoz, I. (2018). Genetic diversity and clonal characteristics of ciprofloxacin-resistant campylobacter jejuni isolated from Chilean patients with gastroenteritis. Infect. Genet. Evol. 58, 290-293. doi: 10.1016/j.meegid.2017.12.026

DAFM (2020). Avian influenza (Bird Flu)- Current Situation: AI Alert 02/2020. Available at: https://www.gov.ie/en/publication/50ce4-avian-influenza-bird-flu/ (Accessed February 4, 2021).

Davies, R., and Wales, A. (2019). Antimicrobial resistance on farms: a review including biosecurity and the potential role of disinfectants in resistance selection. Compr. Rev. Food Sci. Food Saf. 18, 753-774. doi: 10.1111/ 1541-4337.12438

Desmonts, M. H., Dufour-Gesbert, F., Avrain, L., and Kempf, I. (2004) Antimacrobial resistance in campylobacter strains isolated from French broilers before and after antimicrobial growth promoter bans. J. Antimicrob. Chemother. 54, 1025-1030. doi: 10.1093/jac/dkh473

EFSA (2019). The European Union one health 2018 Zoonoses report. EFSA J. 17:e05926. doi: 10.2903/j.efsa.2019.5926

Feldgarden, M., Brover, V., Haft, D. H., Prasad, A. B., Slotta, D. J., Tolstoy, I., et al. (2019). Validating the AMRFinder tool and resistance gene database by using antimicrobial resistance genotype-phenotype correlations in a 
collection of isolates. Antimicrob. Agents Chemother. 63, e00483-e00419. doi: 10.1128/AAC.00483-19

Flanagan, R. C., Neal-McKinney, J. M., Dhillon, A. S., Miller, W. G., and Konkel, M. E. (2009). Examination of campylobacter jejuni putative adhesins leads to the identification of a new protein, designated FlpA, required for chicken colonization. Infect. Immun. 77, 2399-2407. doi: 10.1128/ IAI.01266-08

Frazão, M. R., Medeiros, M. I. C., Da Silva Duque, S., and Falcão, J. P. (2017). Pathogenic potential and genotypic diversity of campylobacter jejuni: a neglected food-borne pathogen in Brazil. J. Med. Microbiol. 66, 350-359. doi: $10.1099 / \mathrm{jmm} .0 .000424$

Gupta, S. K., Padmanabhan, B. R., Diene, S. M., Lopez-Rojas, R., Kempf, M., Landraud, L., et al. (2014). ARG-ANNOT, a new bioinformatic tool to discover antibiotic resistance genes in bacterial genomes. Antimicrob. Agents Chemother. 58, 212-220. doi: 10.1128/AAC.01310-13

Gurevich, A., Saveliev, V., Vyahhi, N., and Tesler, G. (2013). QUAST: quality assessment tool for genome assemblies. Bioinformatics 29, 1072-1075. doi: 10.1093/bioinformatics/btt086

Harvala, H., Rosendal, T., Lahti, E., Engvall, E. O., Brytting, M., Wallensten, A., et al. (2016). Epidemiology of campylobacter jejuni infections in Sweden, November 2011-October 2012: is the severity of infection associated with C. jejuni sequence type? Infect. Ecol. Epidemiol. 6:31079. doi: 10.3402/iee. v6.31079

Hermans, D., Van Deun, K., Martel, A., Van Immerseel, F., Messens, W., Heyndrickx, M., et al. (2011). Colonization factors of campylobacter jejuni in the chicken gut. Vet. Res. 42, 1-14. doi: 10.1186/1297-9716-42-82

HPSC (2020). Epidemiology of COVID-19 in Ireland: March 2020. Available at: https://www.hpsc.ie/a-z/respiratory/coronavirus/novelcoronavirus/casesinireland/ epidemiologyofcovid-19inireland/march2020/(Accessed February 4, 2021).

Huerta-Cepas, J., Forslund, K., Coelho, L. P., Szklarczyk, D., Jensen, L. J., von Mering, C., et al. (2017). Fast genome-wide functional annotation through Orthology assignment by eggNOG-mapper. Mol. Biol. Evol. 34, 2115-2122. doi: $10.1093 / \mathrm{molbev} / \mathrm{msx} 148$

Inglis, G. D., Ramezani, N., Taboada, E. N., Boras, V. F., and Uwiera, R. R. E. (2020). Analysis of campylobacter jejuni subtype distribution in the chicken broiler production continuum: a longitudinal examination to identify primary contamination points. Appl. Environ. Microbiol. 87, e02001-e02020. doi: 10.1128/aem.02001-20

Jia, B., Raphenya, A., Alcock, B., Waglechne, N., Guo, P., and KK, T., et al., (2017). CARD 2017: expansion and model-centric curation of the comprehensive antibiotic resistance database. Nucleic Acids Res. 45:gkw1004. doi: $10.1093 / \mathrm{nar} / \mathrm{gkw} 1004$

Jolley, K. A., Bray, J. E., and Maiden, M. C. J. (2018). Open-access bacterial population genomics: BIGSdb software, the PubMLST.org website and their applications. Wellcome. open. Res. 3:124. doi: 10.12688/wellcomeopenres. 14826.1

Jorgensen, F., Ellis-Iversen, J., Rushton, S., Bull, S. A., Harris, S. A., Bryan, S. J., et al. (2011). Influence of season and geography on campylobacter jejuni and C. coli subtypes in housed broiler flocks reared in Great Britain. Appl. Environ. Microbiol. 77, 3741-3478. doi: 10.1128/AEM.02444-10

Kaakoush, N. O., Castaño-Rodríguez, N., Mitchell, H. M., and Man, S. M. (2015). Global epidemiology of campylobacter infection. Clin. Microbiol. Rev. 28, 687-720. doi: 10.1128/CMR.00006-15

Kim, J. S., Artymovich, K. A., Hall, D. F., Smith, E. J., Fulton, R., Bell, J., et al. (2012). Passage of campylobacter jejuni through the chicken reservoir or mice promotes phase variation in contingency genes Cj0045 and Cj0170 that strongly associates with colonization and disease in a mouse model. Microbiology 158, 1304-1316. doi: 10.1099/mic.0.057158-0

Konkel, M. E., Kim, B. J., Rivera-Amill, V., and Garvis, S. G. (1999). Bacterial secreted proteins are required for the internalization of campylobacter jejuni into cultured mammalian cells. Mol. Microbiol. 32, 691-701. doi: 10.1046/j. 1365-2958.1999.01376.x

Koolman, L., Whyte, P., and Bolton, D. J. (2014). An investigation of broiler caecal campylobacter counts at first and second thinning. J. Appl. Microbiol. 117, 876-881. doi: 10.1111/jam.12580

Lai, C. K., Chen, Y. A., Lin, C. J., Lin, H. J., Kao, M. C., Huang, M. Z., et al. (2016). Molecular mechanisms and potential clinical applications of campylobacter jejuni cytolethal distending toxin. Front. Cell. Infect. Microbiol. 6:9. doi: $10.3389 /$ fcimb.2016.00009
Liu, D., Liu, W., Lv, Z., Xia, J., Li, X., Hao, Y., et al. (2019). Emerging Erm(B)mediated macrolide resistance associated with novel multidrug resistance genomic islands in campylobacter. Antimicrob. Agents Chemother. 63, e00153-e00119. doi: 10.1128/AAC.00153-19

McInerney, J. O., McNally, A., and O'Connell, M. J. (2017). Why prokaryotes have pangenomes. Nat. Microbiol. 2, 1-5. doi: 10.1038/nmicrobiol. 2017.40

Meunier, M., Guyard-Nicodème, M., Dory, D., and Chemaly, M. (2016). Control strategies against campylobacter at the poultry production level: biosecurity measures, feed additives and vaccination. J. Appl. Microbiol. 120, 1139-1173. doi: $10.1111 /$ jam. 12986

Mouftah, S., Cobo-Díaz, J., Álvarez-Ordóñez, A., Mousa, A., Calland, J., Pascoe, B., et al. (2021). Stress resistance associated with multi-host transmission and enhanced biofilm formation at $42^{\circ} \mathrm{C}$ among hyperaerotolerant generalist campylobacter jejuni. Food Microbiol. 95:103706. doi: 10.1016/j.fm.2020.103706

Nielsen, L. N., Sheppard, S. K., McCarthy, N. D., Maiden, M. C. J., Ingmer, H., and Krogfelt, K. A. (2010). MLST clustering of campylobacter jejuni isolates from patients with gastroenteritis, reactive arthritis and Guillain Barre syndrome. J. Appl. Microbiol. 108, 591-599. doi: 10.1111/j.1365-2672. 2009.04444.x

Ocejo, M., Oporto, B., and Hurtado, A. (2019). Occurrence of campylobacter jejuni and campylobacter coli in cattle and sheep in northern Spain and changes in antimicrobial resistance in two studies 10-years apart. Pathogens 8:98. doi: $10.3390 /$ pathogens 8030098

Oh, E., McMullen, L., and Jeon, B. (2015). High prevalence of hyper-Aerotolerant campylobacter jejuni in retail poultry with potential implication in human infection. Front. Microbiol. 6:1263. doi: 10.3389/fmicb.2015.01263

Page, A. J., Cummins, C. A., Hunt, M., Wong, V. K., Reuter, S., Holden, M. T. G., et al. (2015). Roary: rapid large-scale prokaryote pan genome analysis. Bioinformatics 31, 3691-3693. doi: 10.1093/bioinformatics/btv421

Park, C. J., Li, J., Zhang, X., Gao, F., Benton, C. S., and Andam, C. P. (2020). Genomic epidemiology and evolution of diverse lineages of clinical campylobacter jejuni co-circulating in New Hampshire, USA, 2017. J. Clin. Microbiol. 58, e02070-e02019. doi: 10.1128/jcm.02070-19

Prachantasena, S., Charununtakorn, P., Muangnoicharoen, S., Hankla, L., Techawal, N., Chaveerach, P., et al. (2016). Distribution and genetic profiles of campylobacter in commercial broiler production from breeder to slaughter in Thailand. PLoS One 11:e0149585. doi: 10.1371/journal.pone.0149585

Pumbwe, L., and Piddock, L. J. V. (2002). Identification and molecular characterisation of $C m e B$, a campylobacter jejuni multidrug efflux pump. FEMS Microbiol. Lett. 206, 185-189. doi: 10.1111/j.1574-6968.2002.tb11007.x

Rabby, A., Chakraborty, S., Rahman, A., Shakila Rahman, S., Soad, S., Fatima Chanda, K., et al. (2015). Identification of the positively selected genes governing host-pathogen arm race in vibrio sp. through comparative genomics approach Biojournal Sci. Technol. 2:m140008. doi: 10.6084/M9.FIGSHARE.1494598

Sang, F. C., Shane, S. M., Yogasundram, K., Hagstad, H. V., and Kearney, M. T. (1989). Enhancement of campylobacter jejuni virulence by serial passage in chicks. Avian Dis. 33, 425-430. doi: 10.2307/1591100

Sayers, S., Li, L., Ong, E., Deng, S., Fu, G., Lin, Y., et al. (2019). Victors: a web-based knowledge base of virulence factors in human and animal pathogens. Nucleic Acids Res. 47, D693-D700. doi: 10.1093/nar/gky999

Scuron, M. D., Boesze-Battaglia, K., Dlakic, M., and Shenker, B. J. (2016). The cytolethal distending toxin contributes to microbial virulence and disease pathogenesis by acting as a tri-perditious toxin. Front. Cell. Infect. Microbiol. 6:168. doi: $10.3389 /$ fcimb.2016.00168

Seemann, T. (2014). Prokka: rapid prokaryotic genome annotation. Bioinformatics 30, 2068-2069. doi: 10.1093/bioinformatics/btu153

Sgro, G. G., Oka, G. U., Souza, D. P., Cenens, W., Bayer-Santos, E., Matsuyama, B. Y., et al. (2019). Bacteria-killing type IV secretion systems. Front. Microbiol. 10:1078. doi: $10.3389 /$ fmicb.2019.01078

Stamatakis, A. (2014). RAxML version 8: a tool for phylogenetic analysis and post-analysis of large phylogenies. Bioinformatics 30, 1312-1313. doi: 10.1093/ bioinformatics/btu033

Stetsenko, V. V., and Efimochkina, N. R., and Pichugina, T. V (2018). Growth and persistence of campylobacter jejuni in foodstuffs. Eksp. Biol. i Meditsiny 166, 723-730. doi:10.1007/s10517-019-04435-x.

Talukder, K. A., Aslam, M., Islam, Z., Azmi, I. J., Dutta, D. K., Hossain, S., et al. (2008). Prevalence of virulence genes and cytolethal distending toxin 
production in campylobacter jejuni isolates from diarrheal patients in Bangladesh. J. Clin. Microbiol. 46, 1485-1488. doi: 10.1128/JCM.01912-07

Wang, G., Clark, C. G., Taylor, T. M., Pucknell, C., Barton, C., Price, L., et al. (2002). Colony multiplex PCR assay for identification and differentiation of campylobacter jejuni, C. coli, C. lari, C. upsaliensis, and C. fetus subsp. fetus. J. Clin. Microbiol. 40, 4744-4747. doi: 10.1128/JCM.40.12.4744-4747.2002

Wysok, B., Wojtacka, J., Hänninen, M.-L., and Kivistö, R. (2020). Antimicrobial resistance and virulence-associated markers in campylobacter strains From diarrheic and non-diarrheic humans in Poland. Front. Microbiol. 11:1799. doi: $10.3389 /$ fmicb.2020.01799

Yao, H., Shen, Z., Wang, Y., Deng, F., Liu, D., Naren, G., et al. (2016). Emergence of a potent multidrug efflux pump variant that enhances campylobacter resistance to multiple antibiotics. MBio 7, e01543-e01516. doi: 10.1128/ mBio.01543-16

Ziprin, R. L., Young, C. R., Byrd, J. A., Stanker, L. H., Hume, M. E., Gray, S. A., et al. (2001). Role of campylobacter jejuni potential virulence genes in Cecal colonization. Avian Dis. 45, 547-549. doi: 10.2307/1592894
Conflict of Interest: The authors declare that the research was conducted in the absence of any commercial or financial relationships that could be construed as a potential conflict of interest.

Publisher's Note: All claims expressed in this article are solely those of the authors and do not necessarily represent those of their affiliated organizations, or those of the publisher, the editors and the reviewers. Any product that may be evaluated in this article, or claim that may be made by its manufacturer, is not guaranteed or endorsed by the publisher.

Copyright (C) 2021 Truccollo, Whyte, Burgess and Bolton. This is an open-access article distributed under the terms of the Creative Commons Attribution License (CC BY). The use, distribution or reproduction in other forums is permitted, provided the original author(s) and the copyright owner(s) are credited and that the original publication in this journal is cited, in accordance with accepted academic practice. No use, distribution or reproduction is permitted which does not comply with these terms. 